\title{
MORTALIDADE EM BOMBEIROS MILITARES DO ESTADO DO RIO DE JANEIRO - BRASIL: ANÁLISE DE PERÍODO DE DEZ ANOS
}

\author{
Simone Cristina da Silva Coelho ${ }^{1}$ \\ Roberto Kazumi Baldas Miura \\ Luiz Claudio Dias da Rocha \\ Mariana Tschoepke Aires \\ Suzana Tschoepke Aires \\ Melanie Hurel Barroso
}

\section{RESUMO}

Trata-se de um estudo seccional e descritivo cujo objetivo é descrever os dados de mortalidade dos bombeiros militares (BM) do Estado do Rio de Janeiro em um período de 10 anos e comparar com os dados de mortalidade geral da população. Foram calculados os coeficientes e taxas de mortalidade geral e proporcional dos BM e da população do sexo masculino de 2006-2015. A média de idade de óbito dos BM ativos e inativos e as causas foram descritas. Foram identificados 965 óbitos no período. A média de idade do óbito entre Praças e Oficiais foi de $57,0 \pm 12,3$ e $55,5 \pm 13,9$, respectivamente. As doenças cardiovasculares foram as principais causas de óbito e houve um predomínio de causas externas nos bombeiros em atividade. Embora existam poucos estudos sobre o tema em bombeiros militares, observamos alguns dados de mortalidade de BM semelhantes aos da população geral com diferenças significativas nas causas de óbito em bombeiros em atividade. Esses achados demonstram a importância da realização de novos estudos nesses profissionais e a implantação de programas de prevenção de saúde para redução dos óbitos por causas externas.

Palavras-chave: Mortalidade; Medicina Militar; Bombeiros.

\footnotetext{
${ }^{1}$ Corpo de Bombeiros Militar do Estado do Rio de Janeiro. Doutora em Endocrinologia, email: saude.cbmerj@gmail.com
} 


\title{
MORTALITY AMONG MILITARY FIREFIGHTERS OF THE STATE OF RIO DE JANEIRO - BRAZIL: ANALYSIS OVER A 10-YEAR PERIOD
}

\begin{abstract}
This is a cross-sectional, descriptive study that aims is to describe the mortality data of military firefighters of the State of Rio de Janeiro over a period of 10 years and to compare with the general mortality data of the population. The coefficients and general and proportional mortality rates of BM and the male population from 2006-2015 were calculated. The average age of death of active and inactive BM and the causes were described. We identified 965 deaths in the period. The average age of death between Squares and Officers was $57.0 \pm$ 12.3 and $55.5 \pm 13.9$, respectively. Cardiovascular diseases were the main causes of death, and there was a predominance of external causes in active firefighters. Although there are few studies on the subject in military firefighters, we observed some BM mortality data similar to the general population with significant differences in causes of death in active firefighters. These findings demonstrate the importance of further studies on these professionals and the implementation of health prevention programs to reduce the external causes of death.
\end{abstract}

Keywords: Mortality; Military Medicine; Firefighters

Artigo Recebido em 15/01/2020 e Aceito em 08/06/2020 


\section{INTRODUÇÃO}

O Corpo de Bombeiros Militar do Estado do Rio de Janeiro (CBMERJ) é o mais antigo do Brasil, fundado em 02 de julho de 1856. Os Bombeiros Militares (BM) desempenham diversas funções como fiscalização das edificações para a adequação aos padrões exigidos para a prevenção e combate a incêndios, busca e salvamento, prestação de socorros nos casos de inundações, desabamentos, incêndios e catástrofes, acidentes em vias públicas entre outros. Tais atividades são essenciais para a manutenção e equilíbrio da vida em sociedade (Estatuto do Corpo de Bombeiros Militar do Estado do Rio de Janeiro, 1985).

O exercício adequado das funções de BM requer frequentes treinamentos e atualizações. $\mathrm{O}$ BM deve se manter equilibrado emocionalmente, frente ao perfil estressante de suas atividades. Esse cenário de atuação oferece considerável potencial lesivo à sua integridade física e mental. Ampla variedade de doenças vem se manifestando nos BM em decorrência de suas atividades de trabalho, sobretudo doenças osteomusculares, do aparelho circulatório, transtornos mentais e comportamentais. Estes últimos podem ser atribuídos a um ambiente de trabalho intenso e estressante (PIRES, 2016; KNIHS, 2018; FRANCO, 2010; MONTEIRO, 2013; ASSUNÇÃO, 2015).

Embora existam na literatura diversos estudos demonstrando a relação entre agravos de saúde e a atividade de BM, os resultados dos estudos de mortalidade realizados em Corporações Militares são escassos e revelam resultados diferentes dos dados de mortalidade da população geral (PIRES, 2016; MATA, 2017; SILVA, 2000).

O Estado do Rio de Janeiro conta com 12.264 BM (76,7\% Praças e $23,3 \%$ Oficiais); $80,72 \%$ pertencem ao sexo masculino (DGP/CBMERJ, 2020). 
Este efetivo representa o maior em todo território brasileiro, seguido por São Paulo, Minas Gerais e Distrito Federal (DGP/CBMERJ, 2020).

Estudos de mortalidade europeus realizados em militares encontraram uma incidência maior do que o esperado de óbitos por neoplasias de origem digestiva em reto/ânus, pâncreas, faringe, estômago, fígado e na laringe (AMADEO, 2015; PETERSEN, 2018). Em militares norte-americanos observouse maior risco de óbitos por câncer de bexiga, tireoide e mama em homens, sugerindo relação com a exposição ocupacional durante atividades de combate a incêndio. Os autores discutem que estudos adicionais são necessários para investigar esse nexo causal (MA, 2005).

Outros estudos identificaram aumento da mortalidade por câncer de cólon e rim, linfoma não- Hodgkin e mieloma múltiplo. A exposição a certas substâncias como o diesel foi levantada como uma possibilidade de causa para os óbitos nessa categoria profissional (BARIS, 2001).

No Estado do Rio de Janeiro, as doenças do aparelho circulatório foram as causas mais frequentes de morte em 2018, respondendo por cerca de $27,2 \%$ dos óbitos. As neoplasias foram elencadas como a segunda causa mortis seguida pelas causas externas. Em homens, os óbitos por doenças do aparelho circulatório responderam por $26 \%$, enquanto as causas externas e as neoplasias foram responsáveis por $19 \%$ e $15 \%$ da mortalidade, respectivamente. Nas mulheres, $31 \%$ dos óbitos foram ocasionados por doenças cardiovasculares, $17 \%$ por neoplasias e em $12 \%$ foram acarretados por doenças respiratórias (Organização Pan Americana da Saúde, 2002; Ministério da Saúde, 2018).

Estudo realizado em militares da Marinha do Brasil (MB) evidenciou aumento na mortalidade por doenças hepáticas correlacionadas com o uso de álcool (SILVA, 2004). O mesmo grupo de estudo evidenciou nos militares da MB maior associação entre neoplasia de Sistema Nervoso Central e fígado no grupo do Corpo de Saúde, neoplasia de cólon e reto nos Oficiais da Administração e nos Oficiais da Armada e neoplasia de laringe no grupo que 
realizava manutenção e reparos, entretanto a amostra desses estudos foi pequena, limitando a análise (SILVA, 2000).

O presente estudo objetivou determinar as principais causas de óbitos e a idade do óbito entre BM no período de 2006 a 2015.

\section{METODOLOGIA}

Trata-se de um estudo seccional observacional de todos os óbitos de $B M$ do sexo masculino do Estado do Rio de Janeiro (RJ), ocorridos no período de janeiro de 2006 a dezembro de 2015. Foram utilizados dados obtidos nas Declarações de Óbitos da Diretoria Geral de Pessoal, Diretoria de Inativos e Pensionistas e Diretoria Geral de Finanças do CBMERJ e na Secretaria Municipal e Estadual de Saúde do Rio de Janeiro. Foram excluídos do estudo os militares do sexo feminino, cuja amostra era reduzida. Os dados de óbitos nos BM foram comparados com a população geral do RJ no mesmo período. Os dados de óbitos da população foram obtidos na base de dados do DATASUS e a população do estado do Rio de Janeiro no meio do período foi obtida na base de dados do Instituto Brasileiro de Geografia e Estatística-IBGE (MINISTÉRIO DA SAÚDE, 2018; INSTITUTO BRASILEIRO DE GEOGRAFIA E ESTATÍSTICA, 2018).

Quanto aos BM, foram utilizadas as seguintes variáveis: idade de óbito, Posto/Graduação, Militar Ativo/Inativo e causa mortis comparadas com os dados de idade e causa mortis da população geral do sexo masculino no mesmo período.

Os dados foram armazenados e analisados no programa Stata versão 13.0. Foram calculadas as frequências das variáveis estudadas. A análise dos dados foi realizada por meio de estatística descritiva, utilizando-se frequências absolutas e percentuais para as variáveis categóricas, e medidas de tendência central (média) e variabilidade (desvio-padrão e valores máximo e mínimo) 
para as numéricas. Foi calculado o coeficiente geral de mortalidade dos ativos, inativos e da população geral do sexo masculino nos anos de estudo.

Para comparar as proporções de causas de óbitos entre os BM ativos e inativos foi utilizado o teste qui-quadrado, com nível de significância $<0,05$.

Foi calculada a taxa de mortalidade de BM do sexo masculino estratificados pelas seguintes faixas etárias: 18 a 24 anos, 25 a 30 anos, 31 a 35 anos, 36 a 40 anos, 41 a 45 anos, 46 a 50 anos, 51 a 55 anos e acima de 56 anos. Foram calculadas as taxas de mortalidade da população geral do RJ do sexo masculino nos anos de estudo e estratificados pelas seguintes faixas etárias: 20 a 29 anos, 30 a 39 anos, 40 a 49 anos, 50 a 59 anos e mais de 60 anos nos anos do estudo. Optou-se por calcular nesta faixa etária, pois há correspondência com a faixa etária de BM ativos e inativos.

O Coeficiente Geral de Mortalidade (CGM) e as taxas de mortalidade (TM) nos BM e na população do Estado do RJ em cada ano (de 2006 a 2015) foram calculadas pelas seguintes fórmulas:

$\mathrm{CGM}_{\mathrm{BM} \text { ATIVOS}=}$ Número de óbitos de $\mathrm{BM}$ ativos do sexo masculino por todas as causas no período $\mathrm{P} \times 1$.000/Número de $\mathrm{BM}$ ativos do sexo masculino no meio do período $P$

$\mathrm{CGM}_{\text {BM INATIVOS }}$ Número de óbitos de BM inativos por todas as causas no período $\mathrm{P} \times 1.000 /$ Número de BM inativos do sexo masculino no meio do período $P$

CGM POPULAÇÃO SEXO MASCULINO $=$ Número de óbitos da população do sexo masculino por todas as causas no período $\mathrm{P} \times 1.000$ /Número da população do sexo masculino no meio do período $P$

$\mathrm{TM}_{\mathrm{BM} \text { ATIVOS }}=$ Número de óbitos de BM ativos do sexo masculino por todas as causas na faixa etária e ano x 100.000/Número de BM ativos do sexo masculino por ano e faixa etária 
$\mathrm{TM}_{\text {BM INATIVOS }}=$ Número de óbitos de BM inativos por todas as causas na faixa etária e ano x 100.000/ Número de BM inativos do sexo masculino por ano e faixa etária

TM POPULAÇÃO SEXO MASCULINO = Número de óbitos da população do sexo masculino por todas as causas estratificadas por idade e ano $\times 100.000 /$ Número da população do sexo masculino por ano

O trabalho foi aprovado pelo Comitê de Ética em Pesquisa do Instituto Estadual de Cardiologia Aloysio de Castro/ SES (Parecer número 2.500.968 de 19/02/2018).

\section{RESULTADOS}

No período de estudo registraram-se 965 óbitos de BM do sexo masculino. Na população masculina acima de 20 anos no estado do RJ foram registrados 754.244 óbitos nesse mesmo período. Os óbitos dos BM foram classificados como aqueles que faleceram enquanto estavam ativos no serviço militar e os que estavam inativos por reserva ou reforma (Tabela1). A idade de

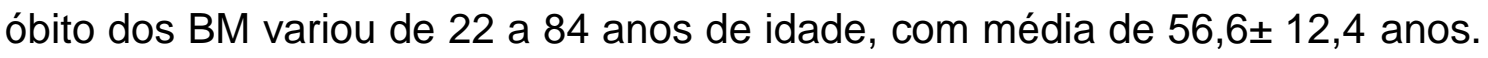
Os óbitos ocorreram em $91,5 \%$ de Praças e $8,5 \%$ de Oficiais. A tabela 1 resume as características dos óbitos entre BM.

A média de idade do óbito entre Praças foi de $57,0 \pm 12,3$, variando de 22 a 84 anos. Entre os oficiais a média de idade de óbito foi $55,5 \pm 13,9$, variando de 23 a 80 anos. A média de idade de óbito no grupo de ativos foi de $42,6 \pm 8,2$ (variando de 22 a 63 anos). No grupo de inativos, a média foi de 63 $\pm 8,0$ (variando de 39 a 84 anos). 
Tabela1 - Distribuição dos óbitos segundo idade, posto, local, ano e situação do BM (ativo ou inativo) no período de 2006 a 2015.

\begin{tabular}{|c|c|c|}
\hline Características dos óbitos entre os BM (N=965) & $\mathbf{N}$ & $\%$ \\
\hline \multicolumn{3}{|l|}{ Posto } \\
\hline Praça & 883 & $91,5 \%$ \\
\hline Oficial & 82 & $8,5 \%$ \\
\hline \multicolumn{3}{|l|}{ Situação } \\
\hline Ativo & 229 & $31 \%$ \\
\hline Inativo & 666 & $69 \%$ \\
\hline \multicolumn{3}{|l|}{ Idade do óbito } \\
\hline 18 a 24 anos & 05 & $0,5 \%$ \\
\hline 25 a 30 anos & 23 & $2,4 \%$ \\
\hline 31 a 35 anos & 38 & $3,9 \%$ \\
\hline 36 a 40 anos & 51 & $5,3 \%$ \\
\hline 41 a 45 anos & 78 & $8,1 \%$ \\
\hline 46 a 50 anos & 78 & $8,1 \%$ \\
\hline 51 a 55 anos & 149 & $15,4 \%$ \\
\hline acima de 56 anos & 543 & $56,3 \%$ \\
\hline \multicolumn{3}{|l|}{ Local do óbito } \\
\hline Região Metropolitana do Estado do RJ & 754 & $78,1 \%$ \\
\hline Interior do Estado do RJ & 106 & $11 \%$ \\
\hline Fora do Estado do RJ & 15 & $1,6 \%$ \\
\hline Ignorado & 90 & $9,3 \%$ \\
\hline \multicolumn{3}{|l|}{ Ano do óbito } \\
\hline 2006 & 85 & $8,7 \%$ \\
\hline
\end{tabular}


Revista Científica do Corpo de Bombeiros Militar de Pernambuco

Artigo Publicado no Vol.06 N.16 - Edição Especial 2020 - ISSN 2359-4829

Versão on-line disponível em: http://www.revistaflammae.com

\begin{tabular}{clll}
\hline 2007 & 92 & $9,5 \%$ \\
2008 & 79 & $8,2 \%$ \\
2009 & 103 & $10,6 \%$ \\
2010 & 113 & $11,8 \%$ \\
2011 & 90 & $9,3 \%$ \\
2012 & 98 & $10,2 \%$ \\
2013 & 106 & $11 \%$ \\
& 2014 & 98 & $10,2 \%$ \\
& 2015 & 101 & $10,5 \%$ \\
\hline Total & & 965 & $100 \%$ \\
\hline
\end{tabular}

Tabela 2 - Causa de morte entre BM ativos, inativos e população geral do sexo masculino no Estado do RJ (2006 a 2015).

\begin{tabular}{lllll}
\hline Causa & $\begin{array}{l}\text { BM } \\
\text { ativos }\end{array}$ & $\begin{array}{l}\text { BM } \\
\text { inativos }\end{array}$ & Total BM & População \\
\hline Doenças cardiovasculares & $16,4 \%$ & $35,8 \%^{*}$ & $29,7 \%$ & $27,6 \%$ \\
\hline Causas Externas & $48,5 \%$ & $6,6 \% \%^{*}$ & $19,6 \%$ & $11,3 \%$ \\
\hline Neoplasias & $8,4 \%$ & $17,7 \% *$ & $14,8 \%$ & $14,4 \%$ \\
\hline Doenças Infecciosas & $9,0 \%$ & $15,5 \%$ & $13,6 \%$ & $5,3 \%$ \\
\hline Causas Indeterminadas & $6,3 \%$ & $5,1 \%$ & $5,5 \%$ & $7,9 \%$ \\
\hline Causas Metabólicas & $1,7 \%$ & $5,6 \%$ & $4,3 \%$ & $5,3 \%$ \\
\hline Outras & $9,7 \%$ & $13,7 \%$ & $12,5 \%$ & $28,2 \%$ \\
\hline Total & $100 \%$ & $100 \%$ & $100 \%$ & $100 \%$ \\
\hline
\end{tabular}

${ }^{*} \mathrm{p}<0,05$.

A principal causa de morte foi doença cardiovascular, que representou 29,7\% dos óbitos (IC 95\%: 26,9-32,7\%), variando de 16,4\% entre os ativos a 
Revista Científica do Corpo de Bombeiros Militar de Pernambuco

Artigo Publicado no Vol.06 N.16 - Edição Especial 2020 - ISSN 2359-4829

Versão on-line disponível em: http://www.revistaflammae.com

$35,8 \%$ entre os inativos. As causas externas representam a segunda maior causa de morte, compreendendo $19,6 \%$ do total de óbitos (IC95\%:17,1 a $22,2 \%)$. As causas externas predominaram entre os BM em atividade, representando $48,5 \%$ de todas as causas (IC95\%: 42,7 a $54,9 \%$ ).

Houve diferença significativa entre óbitos por doenças cardiovasculares, causas externas e neoplasias entre ativos e inativos. Quanto às demais causas, não houve diferença estatisticamente significativa.

A tabela 3 mostra o coeficiente de mortalidade dos BM ativos, inativos e da população geral. As taxas de mortalidade geral por faixa etária dos BM e da população geral estão descritas nas tabelas 4 e 5.

Tabela 3 - Coeficiente de mortalidade dos BM ativos, BM inativos e da população geral do sexo masculino nos anos de estudo.

\begin{tabular}{lllc}
\hline Ano & Ativos & Inativos & População Geral \\
\hline 2006 & 230 & 1140 & 1200 \\
\hline 2007 & 180 & 1450 & 1200 \\
\hline 2008 & 160 & 1210 & 1200 \\
\hline 2009 & 300 & 1700 & 1190 \\
\hline 2010 & 270 & 1420 & 1210 \\
\hline 2011 & 200 & 1160 & 1180 \\
\hline 2012 & 150 & 1390 & 1150 \\
\hline 2013 & 180 & 1350 & 1180 \\
\hline 2014 & 170 & 1240 & 1160 \\
\hline 2015 & 90 & 1350 & 1150 \\
\hline
\end{tabular}


Tabela 4 - Taxa de mortalidade dos BM ativos do sexo masculino estratificadas por idade por 100.000 por todas as causas, entre 2006 e 2015 .

\begin{tabular}{lllllllll}
\hline Ano & $\mathbf{1 8}$ a 24 & $\mathbf{2 5}$ a 30 & $\mathbf{3 1}$ a 35 & $\mathbf{3 6}$ a $\mathbf{4 0}$ & $\mathbf{4 1}$ a $\mathbf{4 5}$ & $\mathbf{4 6}$ a 50 & $\mathbf{5 1}$ a 55 & $\begin{array}{l}\mathbf{5 6} \text { ou } \\
\text { mais }\end{array}$ \\
\hline 2006 & 111 & 220 & 130 & 150 & 280 & 550 & 150 & 540 \\
\hline 2007 & 0 & 60 & 200 & 120 & 250 & 430 & 810 & 0 \\
\hline 2008 & 0 & 0 & 220 & 130 & 190 & 250 & 470 & 122 \\
\hline 2009 & 0 & 80 & 200 & 260 & 480 & 240 & 148 & 0 \\
\hline 2010 & 211 & 220 & 100 & 330 & 260 & 450 & 400 & 350 \\
\hline 2011 & 130 & 50 & 180 & 130 & 160 & 390 & 410 & 340 \\
\hline 2012 & 0 & 120 & 0 & 200 & 220 & 130 & 300 & 121 \\
\hline 2013 & 0 & 140 & 160 & 30 & 260 & 200 & 400 & 173 \\
\hline 2014 & 0 & 80 & 140 & 200 & 160 & 180 & 180 & 520 \\
\hline 2015 & 0 & 0 & 0 & 0 & 170 & 170 & 160 & 0 \\
\hline
\end{tabular}

Tabela 5 - Taxa de mortalidade da população do sexo masculino do Estado do Rio de Janeiro, estratificada por idade por 100.000 por todas as causas, entre

\begin{tabular}{lllllc}
\multicolumn{7}{c}{2006 e 2015. } \\
\hline Ano & 20 a 29 & 30 a 39 & 40 a 49 & 50 a 59 & Acima de 60 \\
\hline 2006 & 350 & 350 & 600 & 1280 & 4950 \\
\hline 2007 & 370 & 340 & 600 & 1290 & 4840 \\
\hline 2008 & 330 & 330 & 580 & 1270 & 4890 \\
\hline 2009 & 350 & 330 & 560 & 1230 & 4710 \\
\hline 2010 & 300 & 310 & 540 & 1240 & 4830 \\
\hline 2011 & 280 & 310 & 530 & 1190 & 4630 \\
\hline 2012 & 270 & 300 & 490 & 1140 & 4440 \\
\hline
\end{tabular}


Revista Científica do Corpo de Bombeiros Militar de Pernambuco

Artigo Publicado no Vol.06 N.16 - Edição Especial 2020 - ISSN 2359-4829

Versão on-line disponível em: http://www.revistaflammae.com

\begin{tabular}{llllll}
\hline 2013 & 280 & 300 & 510 & 1130 & 4450 \\
\hline 2014 & 300 & 290 & 470 & 1080 & 4310 \\
\hline 2015 & 290 & 270 & 450 & 1050 & 4240 \\
\hline
\end{tabular}

A média de idade no momento dos óbitos por doenças cardiovasculares e causas externas entre todos os BM (ativos e inativos) foi de 60,4 $\pm 9,3$ anos (variando de 30 a 78 anos) e $44.1 \pm 12.6$ anos (variando de 22 a 84 anos) respectivamente no período de 2006 a 2015.

\section{DISCUSSÃo}

Até onde temos conhecimento, este é o primeiro estudo que caracteriza vários aspectos relacionados à mortalidade de BM no Brasil. Pela escassez de dados referentes a esse assunto, tanto no Brasil quanto em outros países da América Latina, é muito importante o conhecimento dessas taxas de mortalidade na nossa população, as circunstâncias do evento e as características das vítimas, para que estratégias de prevenção sejam desenvolvidas e testadas, algumas delas específicas da categoria profissional.

Muitos estudos sobre mortalidade entre Bombeiros foram conduzidos em diferentes países, entre eles os Estados Unidos, França e Dinamarca e as causas de mortalidade foram semelhantes (KAHN, 2019; MA, 2005; EMUEGGE, 2018; AMADEO, 2015 e PETERSEN, 2018).

A principal causa de morte entre BM e na população geral no presente estudo no período de 10 anos foi representada pelas doenças cardiovasculares o que se assemelha ao observado no estudo de Kahn e colaboradores (2019). Vale ressaltar, no entanto, que quando avaliamos os BM em atividade houve um predomínio das causas externas. Tais resultados são preocupantes, pois 
esta categoria profissional apresenta inúmeras peculiaridades e envolve diversos riscos na sua execução laboral.

Khan e colaboradores (2019), ao comparar a mortalidade de bombeiros em dois momentos distintos (1990-2009 e 2010-2016), verificaram que a mortalidade por doenças cardiovasculares manteve-se constante, no entanto a mortalidade por trauma diminuiu nos períodos estudados. Embora nossa casuística seja menor, o presente trabalhou evidenciou que a mortalidade por causas externas no grupo de $\mathrm{BM}$ em atividade manteve-se constante ao longo de uma década. Esse achado reforça que a prevenção de acidentes e óbitos por traumas entre os BM deve ser valorizada e considerada um aspecto primordial na formação desses profissionais ao longo da carreira militar.

Em Indiana, EUA, verificou-se que os Bombeiros possuem maior chance de falecer de doenças neoplásicas quando comparados à população geral (EMUEGGE, 2018). Verificamos no nosso estudo que as neoplasias figuram como segunda causa de óbito com predomínio nos BM inativos. Trata-se de um aspecto relevante, pois a nossa casuística envolveu um número maior de $\mathrm{BM}$ inativos e os achados foram semelhantes à população geral no mesmo período.

Outro tema importante que deve ser considerado é o efeito do trabalhador saudável. Na França, Amadeo e colaboradores (2015) observaram menor taxa de mortalidade entre os Bombeiros, em virtude do efeito do trabalhador saudável.

Trata-se, no entanto, de tema controverso porque o efeito do trabalhador saudável não é um evento uniforme quando são analisadas diversas variáveis como sexo, idade, etnia e ocupação. Essa variabilidade suscita uma análise estatística com métodos sofisticados, o que dificulta a sua comparação com os dados estudados (CHOWDHURY, 2017). 


\section{CONCLUSÃO}

Neste trabalho observamos que alguns dados de mortalidade dos BM se assemelham aos da população geral, contudo com diferenças ao serem considerados os BM em atividade. No entanto, existe um número reduzido de publicações para comparação nesse grupo específico em nosso meio, o que evidencia a relevância de novos estudos específicos de mortalidade envolvendo BM.

Destaca-se que a saúde dos BM é um recurso extraordinário para a eficácia no seu desempenho e para a sua qualidade de vida. Os dados encontrados apontam a necessidade da implantação de programas de prevenção e promoção à saúde na Corporação com o objetivo de reduzir os percentuais elevados de mortalidade por causas externas, doenças cardiovasculares e neoplasias nessa categoria profissional. Estudos prospectivos adicionais são necessários para melhor caracterização dos óbitos entre os BM no nosso meio.

\section{REFERÊNCIAS}

AMADEO, B. et al. French firefighter mortality: analysis over a 30-year period. Am J Ind Med, v. 58, n.4, 437-43, 2015.

BARIS, D. et al. Cohort mortality study of Philadelphia firefighters. Am J IndMed, v.39, n.5, 463-76, 2001.

BRASIL. Instituto Brasileiro de Geografia e Estatística- Base de dados do IBGE. Disponível em: http://www.ibge.gov.br/estatisticas/sociais/populacao/9103-estimativas-depopulacao.html?=\&t=o-que-e. Acesso em 10 de outubro de 2018.

. Ministério da Saúde. DATASUS. Informações em Saúde. Mortalidade Rio de Janeiro. Causa CID-BR 10. Período: 2018. Disponível em:http:/tabnet.datasus.gov.br/cgi/tabcgi.exe?sim/cnv/obt10rj.def. Acesso em 09 de outubro de 2019. 
ORGANIZAÇÃO PAN AMERICANA DA SAÚDE. Perfis de saúde e de mortalidade no Brasil:uma análise de seus condicionantes em grupos populacionais específicos. Brasília: OPAS/OMS;2002.

CHOWDHURY, R. et al. Healthy Worker Effect Phenomenon: Revisited with Emphasis on Statistical Methods - A Review. Indian J Occup Environ Med, v.21, n. 1, 2-8, 2017.

CORPO DE BOMBEIROS MILITAR DO ESTADO DO RIO DE JANEIRO, Diretoria Geral de Pessoal. Disponível em:http://www.cbmerj.rj.gov.br/dgp/acesso.php. Acesso em 20 de maio de 2016.

FRANCO, T. et al. As novas relações de trabalho, o desgaste mental do trabalhador e os transtornos mentais no trabalho precarizado. RevBrasSaudeOcup, v. 35, n. 122,229-248, 2010.

KAHN, S.A. et al. Crude Mortality Rates and Risk Factors for Line of Duty Injury and Death.Journal of Burn Care \& Research, v.40, n.2, 196-201, 2019.

KNIHS, D.A. et al. Perfil antropométrico de bombeiros militares: comparação entre os grupos de trabalho operacional e administrativo. RevBrasMedTrab,v.16, n.1, 19-25, 2018.

LIMA, E.P. et al. Transtorno de Estresse Pós-Traumático (TEPT) em Bombeiros de Belo Horizonte, Brasil: Prevalência e Fatores Ocupacionais Associados. Psicologia: Teoria e Pesquisa, v. 31, n. 2, 279-288, 2015.

MA, F. et al. Mortality in Florida professional firefighters, 1972 to 1999. Am J Ind Med, v. 47, n.6, 509-17, 2005.

MATA, N.T. et al. Bombeiros militares: um olhar sobre a saúde e violência relacionados com o trabalho. Saúde Debate, v.41, n.112, 133-141, 2017.

MONTEIRO, J.K. et al. Bombeiros: psicopatologia e condições de trabalho. Estudos de Psicologia, v.30, n.3, 437-444, 2013.

EMUEGGE, C.M. et al. Excess mortality among Indiana firefighters,1985-2013. Am J Ind Med, v.61, n.12, 961-967, 2018.

PETERSEN, K.U et al. Mortality in a cohort of Danish firefighters 1970-2014.Int Arch Occup Environ Health, v. 91, n.6, 759-766, 2018. 


\section{Revista FLAMMAE}

Revista Científica do Corpo de Bombeiros Militar de Pernambuco

Artigo Publicado no Vol.06 N.16 - Edição Especial 2020 - ISSN 2359-4829

Versão on-line disponível em: http://www.revistaflammae.com

PIRES, L.A.A. A relação saúde-trabalho dos bombeiros militares do município do Rio de Janeiro. 2016. 210 f. Dissertação (Mestrado em Saúde Pública) - Escola Nacional de Saúde Pública Sergio Arouca, Fundação Oswaldo Cruz, Rio de Janeiro.

Rio de Janeiro. Lei no 880 de 25 de julho de 1985. Estatuto do Corpo de Bombeiros Militar do Estado do Rio de Janeiro. 1985. Disponível em: http://aleriln1.aleri. Acesso em 10 de outubro de 2019.

SILVA, M. e SANTANA, V.S. Ocupação e mortalidade na Marinha do Brasil. Rev Saúde Pública, v.38, n.5, 709-15, 2004.

SILVA, M. et al. Mortalidade por câncer em militares da Marinha do Brasil. Rev Saúde Pública, v.34, n.4, 373-79, 2000. 